\title{
评 述厦门大学百年校庆物理天文专辑
}

\section{钙离子信号及细胞调控信号网络动力学}

\author{
李翔 ${ }^{1}$, 祁宏 $^{2}$, 黄艳东 $^{3}$, 帅建伟 ${ }^{1 *}$ \\ 1. 厦门大学物理科学与技术学院物理学系, 生物仿生与软物质研究院, 福建省柔性功能材料重点实验室, 厦门 361005 ; \\ 2. 山西大学复杂系统研究所, 太原 030006; \\ 3. 集美大学计算机工程学院, 厦门 361021 \\ *联系人, E-mail: jianweishuai@xmu.edu.cn
}

收稿日期: 2020-11-02; 接受日期: 2020-12-21; 网络出版日期: 2021-02-01

国家自然科学基金(编号: 11675134, 11704318, 11874310)资助项目

摘要钙离子 $\left(\mathrm{Ca}^{2+}\right)$ 是细胞内广泛存在的一种重要的第二信使, 参与并控制着几乎所有的生命活动过程. 细胞信 号分子网络对细胞正常和病理生理活动过程进行着精密调控, 确保细胞各项生理功能有序地进行. 本文综述了近 些年本课题组关于细胞内钙信号及细胞信号网络动力学模型方面的研究进展, 包括集团化钙离子通道释放局域 钙信号、细胞全局钙波信号、内质网和线粒体钙微域调控钙信号和钻信号调控细胞调亡信号网络动力学, 以及 细胞信号调控网络动力学等. 这些理论工作为研究钙信号和蛋白质信号网络调控细胞复杂生命过程的动力学机 制提供了方向和思路.

关键词钙信号, 内质网, $\mathrm{IP}_{3} \mathrm{R}$ 离子通道, 细胞死亡, 信号网络动力学

PACS: 87.16.Vy, 87.50.cf, 87.17.Aa

\section{1 引言}

细胞是生命体的基本结构和功能单元，钙离子 $\left(\mathrm{Ca}^{2+}\right)$ 则是细胞内广泛存在的一种重要的胞内信使, 几乎所有细胞的生理活动都受 $\mathrm{Ca}^{2+}$ 的调控, 例如肌细 胞的收缩, 神经递质从神经元和星形胶质细胞的释放, 受精卵的激活，肝脏和胰腺创伤的愈合和新陈代谢过 程, 以及细胞的成熟、分化和死亡等 ${ }^{[1-4]}$.

钙信号的作用机制既有简单的一面，又有复杂的 一面. 其简单性体现在仅依靠细胞内 $\mathrm{Ca}^{2+}$ 浓度的提升, 便可控制细胞活动. 钲信号其实是细胞内 $\mathrm{Ca}^{2+}$ 浓度的
短暂升高, 它可以把细胞表面接收到的胞外信息传达 到细胞内部的目标分子, 或者通过细胞内部的通讯机 制把信息传达给周围的一组细胞，其信息通常是以钙 波的形式来编码和传递. 钙信号的复杂性体现在它可 以调控细胞的多种生理活动 ${ }^{[2]}$. 这种复杂性来源于细 胞内有多种钙调控蛋白, 包括多种 $\mathrm{Ca}^{2+}$ 通道、 $\mathrm{Ca}^{2+}$ 原、 $\mathrm{Ca}^{2+}$ 转运体、 $\mathrm{Ca}^{2+}$ 交换体和 $\mathrm{Ca}^{2+}$ 结合蛋白等, 这些 蛋白控制着 $\mathrm{Ca}^{2+}$ 的流动过程, 它们之间的不同组合产 生了拥有各种不同时空动力学特性的钻信号. 细胞通 过对 $\mathrm{Ca}^{2+}$ 在时间和空间的浓度振荡图案进行编码构成 钙信号, 这种信息编码形式是根据细胞 $\mathrm{Ca}^{2+}$ 动力学所

引用格式: 李翔, 祁宏, 黄艳东, 等. 铝离子信号及细胞调控信号网络动力学. 中国科学: 物理学 力学 天文学, 2021, 51: 030010

Li X, Qi H, Huang Y D, et al. Dynamics of calcium signals and the intracellular regulatory signaling network (in Chinese). Sci Sin-Phys Mech Astron, 2021, 51: 030010, doi: 10.1360/SSPMA-2020-0442 
产生的多种多样的空间和时间结构来实现的，包括局 部随机释放的钙脉冲 ${ }^{[5]}$ 和全局钻离子振荡波 ${ }^{[6]}$ 等. 钙 离子振荡的周期可以从几秒到几小时，振荡形状可以 从简单的周期振荡到复杂的混沌行为 ${ }^{[7]}$. 目前发现的 钙信号波形有螺旋波 ${ }^{[8]}$ 、靶波 ${ }^{[9]}$ 或介导稳态过渡的锋 面波 ${ }^{[10,11]}$, 展示了诸多非平衡动力学系统的时空结构 特性.

细胞溶质中钙信号的调控, 主要通过细胞钙库中 $\mathrm{Ca}^{2+}$ 摄取或释放进行控制，内质网和线粒体是细胞内 的最主要钙库，在钙信号中起着极其重要的作用。当 前研究认为细胞内的钻稳态主要是通过内质网来保持 的，它可以存储大部分的钙离子 ${ }^{[12]}$. 当细胞受到诸如 荷尔蒙、生长因子、神经递质以及光等刺激时，会通 过磷酸肌醇途径产生肌醇 $1,4,5$-三磷酸 $\left(\mathrm{IP}_{3}\right)$ 分子，调控 内质网膜上 $\mathrm{IP}_{3}$ 受体钙离子通道 $\left(\mathrm{IP}_{3} \mathrm{R}\right)$, 将 $\mathrm{IP}_{3}$ 分子信号 转化为 $\mathrm{Ca}^{2+}$ 信号 ${ }^{[13]}$.

研究表明, $\mathrm{IP}_{3} \mathrm{R}$ 钙离子通道在内质网膜上呈小集 团分布，每个集团内约有十几或二三十个 $I_{3} \mathrm{R}$ 通道， 每个集团空间尺度约几百纳米，集团与集团之间的距 离约几个微米 ${ }^{[14-18]}$. $\mathrm{IP}_{3} \mathrm{R}$ 通道的小集团分布，导致多 尺度的钙离子信号现象(图1)：通过单个 $\mathrm{IP}_{3} \mathrm{R}$ 通道所释 放进细胞溶质的局域瞬时钙离子信号称为钙脉冲
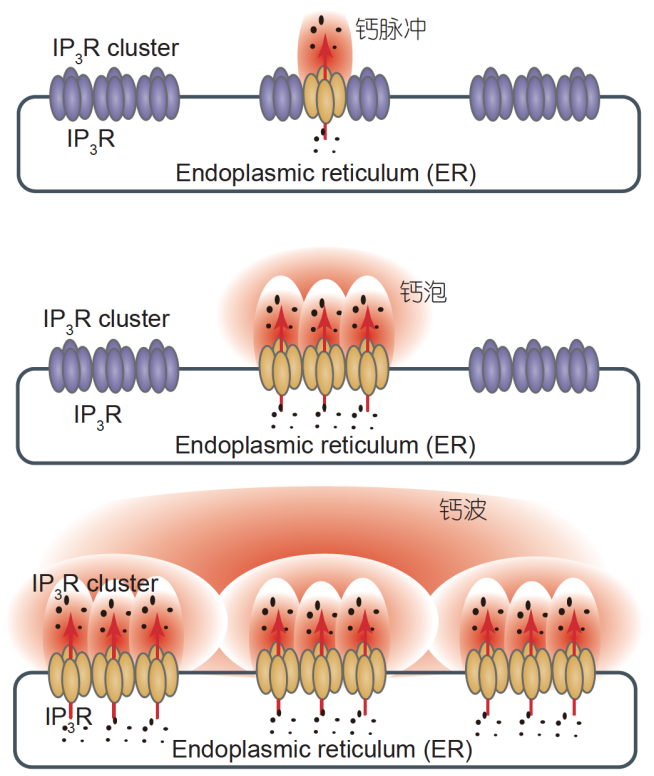

图 1 (网络版彩图)内质网钙离子释放诱发的钻脉冲、钻泡 及钻波等不同信号的示意图

Figure 1 (Color online) Schematic diagram of $\mathrm{Ca}^{2+}$ blip, puff and wave induced by the release of $\mathrm{Ca}^{2+}$ from endoplasmic reticulum.
(Blip), 其自由钻离子仅在几十个纳米的局域范围呈高 浓度分布，其平均寿命约几个微秒 ${ }^{[15]}$; 通过单个小集 团通道所释放的局域瞬时钙离子信号称为钙泡(Puff), 其自由钙离子仅在几百个纳米的局域范围呈高浓度分 布, 其平均寿命约一百个微秒 ${ }^{[5,14-16]}$; 钙离子信号在细 胞溶质内大面积微米尺度的释放称为钲波(Wave), 其 波动的周期通常长达秒和分钟的量级. 局部和整体的 钙信号调节着不同尺度范围的细胞生理功能.

线粒体则是一个临时储存 $\mathrm{Ca}^{2+}$ 的钙库, $\mathrm{Ca}^{2+}$ 通过 线粒体内膜上的线粒体钙单向转运体(MCU)暂时性地 进入线粒体. 虽然线粒体钙摄钻现象在1961年就已经 被发现，但关于线粒体钙信号的主要研究直到世纪之 交才蹒跚起步. 造成此局面的一个重要原因是研究者 们发现MCU对 $\mathrm{Ca}^{2+}$ 的亲和力太低, 据此推断在正常生 理情况下线粒体并不能摄入太多的 $\mathrm{Ca}^{2+}$, 线粒体摄钲 仅在病理状态下发挥作用. 1993年由Rizzuto等人 ${ }^{[19]}$ 提 出的“钲微域假说”促使该领域发生了重大的进步。该 假说认为, 处于开放状态的 $\mathrm{IP}_{3} \mathrm{R}$ 通道口会形成一个高 钙微域，可以满足MCU的摄钙需求. 随后便有很多实 验证据表明线粒体与内质网之间距离极近 ${ }^{[20-22]}$ ，间接 支持了该假说. 2010年的实验证明钙微域在活细胞内 确实存在 ${ }^{[23,24]}$ : 细胞在受到正常生理刺激时, $\mathrm{IP}_{3} \mathrm{R}$ 通道 周围会形成一个高钲微域, 这一微域正好可被对 $\mathrm{Ca}^{2+}$ 低亲和力的MCU所感知, 使得线粒体在正常生理状态 下也可以摄取 $\mathrm{Ca}^{2+}$.2011年MCU的分子鉴定 ${ }^{[25]}$ 使得围 绕线粒体钙信号的研究至今方兴未艾 ${ }^{[26]}$.

进一步，细胞通过调控蛋白分子信号网络的时空 动力学来控制信号的传导, 对细胞各种正常和病理功 能进行调控，包括对基因表达、存活增殖、新陈代 谢、分泌、肌肉收缩、学习记忆、细胞衰老和死亡以 及各种应激响应等，确保细胞各项生理功能有序地进 行. 为了更好地理解细胞信号网络的结构与功能, 物 理学及其交叉学科研究者利用多种理论和方法从不同 角度来研究蛋白质信号网络的结构和调控机制。早期 薛定谔提出的“负熵”观点对后续研究影响深远; 近期 人们通过对细胞信号网络进行理论建模, 开展定量计 算和动力学分析研究, 并发展了一系列理论和方法. 例如，基于分子间的相互作用进行建模，运用化学反 应动力学来描述和刻画细胞信号网络的动态演化过 程; 利用非线性动力学理论如分岔和稳定性分析, 研究 网络系统对各类信号响应的稳态特性; 基于随机微分 
方程，用能量景观和能量耗散理论来刻画系统的全局 稳定性、鲁棒性和敏感性等.

本文主要围绕 $I P_{3} R$ 钙离子通道调控的钙信号, 以 及细胞调控信号网络动力学建模分析, 回顾总结了近 年来本课题组在该领域的研究进展. 我们从内质网上 的单个 $\mathrm{IP}_{3} \mathrm{R}$ 钙离子通道模型出发, 依次介绍了局域钻 脉冲和钙泡信号、全局钙波信号，进而到内质网与线 粒体间的微域钙信号模型的研究, 最后拓展到钻信号 和细胞蛋白信号网络如何参与调控疾病及细胞死亡等 动力学研究.

\section{2 内质网 $I P_{3} R$ 通道扩散动力学}

对于钙信号的研究，相当一部分集中在 $I_{3} \mathrm{R}$ 通道 表达的铛信号时空动力学. 实验发现, $\mathrm{IP}_{3} \mathrm{R}$ 通道在内 质网膜上是可以扩散的，它们可以根据细胞刺激动态 迁移 ${ }^{[27-30]}$. 因此, 我们分别用诺依曼函数解析求解和 蒙特卡罗随机模拟，计算了扩散的通道对膜片上的吸 引域的空间平均首通时间(MFPT)动力学性质.

我们考虑一块有限面积的内质网膜片，在膜片上 含有一个可以针定通道的小吸引区域 ${ }^{[31]}$. 研究发现, 随着通道半径的增加，必须考虑当反弹边界条件应用 时, 扩散通道只在一个有效面积内行走. 这时, MFPT 的理论模型要扩展为有限尺度粒子模型。如果吸引域 的位置保持在离区域中心的距离为半个区域特征长度 范围内，总面积相同的圆形区域和方形区域的空间平 均MFPT是一样的(图2). 研究表明, 在只需要一个长度 尺度来表征的对称区域中，如果吸引域距离区域中心 足够近，它的空间平均MFPT对膜片边界的形状是不 敏感的. 当吸引域在区域中心，对于相同面积的不同 形状的区域, 方形区域的空间平均MFPT最小.

\section{3 内质网 IP ${ }_{3} R$ 调控的局域钻信号动力学}

在内质网膜上十几个 $I_{3} R$ 通道可聚集在一起成团 簇分布 ${ }^{[15]}$, 成团的 $I P_{3} R$ 表现出强烈的随机开关动力学, 会导致复杂的 $\mathrm{Ca}^{2+}$ 信号. 起初是一个单通道打开, 释放 出钙脉冲，接下来导致该单通道附近的几个通道通过 钙诱发钙释放，于是这个团簇中的几个通道打开，释 放出钙泡，形成钙信号的基本事件 ${ }^{[32]}$. 针对局域钙信 号具有很强的随机释放行为, 我们利用随机通道开关

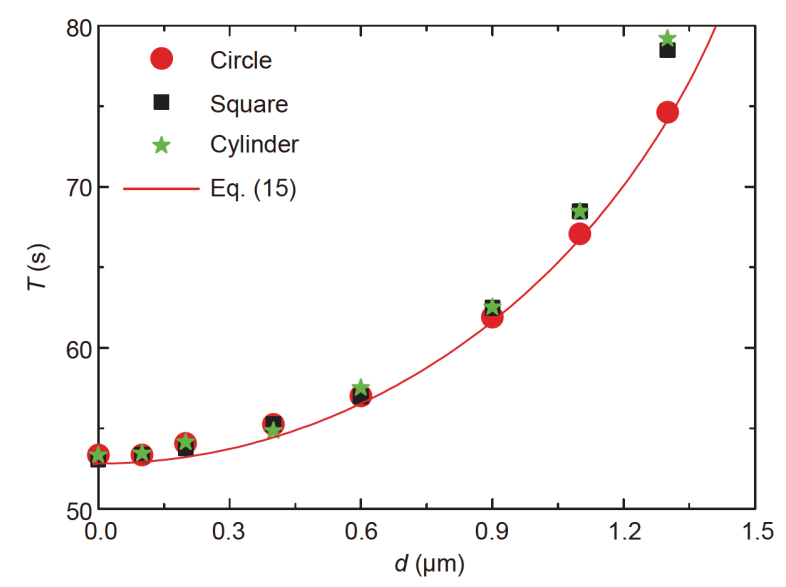

图 2 (网络版彩图)不同几何形状区域的空间平均MFPT和 吸引域的位置到区域中心的距离 $d$ 的依赖关系图 ${ }^{[31]}$

Figure 2 (Color online) Dependence of the average MFPT on the distance $d$ of the anchor site from the patch center for different domain shapes [31].

动力学模型, 深入分析了钙脉冲和钲泡的随机动力学 性质, 及其可能的调控功能作用.

\section{1 钙通道集团调控的局域钙信号动力学}

早期人们就已提出一些模型来研究单个 $\mathrm{IP}_{3} \mathrm{R}$ 通道 释放钻脉冲的动力学行为 ${ }^{[33,34]}$. 我们基于已经发表的 Rüdiger-Shuai模型 ${ }^{[35]}$, 进一步考虑生理条件下 $\mathrm{Ca}^{2+}$ 结 合蛋白等因素的影响, 研究了人类成神经细胞瘤 SHSY5Y细胞中的局域钙信号动力学 ${ }^{[36]}$.

研究指出, 由于受 $\mathrm{IP}_{3}$ 和 $\mathrm{Ca}^{2+}$ 随机结合/解离动力学 的影响, 钙通道集团内第一个开放的钙通道, 既可诱导 集团内其他通道打开而产生钙泡，又可能关闭而仅产 生钙脉冲，这种竞争机制决定了通道集团中局域钻释 放动力学呈现出复杂的模式. 我们发现相邻局域释放 事件之间的时间间隔以及第一次释放事件的响应延迟 时间与通道集团中通道数目的倒数呈线性关系. 在 SH-SY5Y这种体积较小且通道数目较少的细胞中, 钙 脉冲可能会通过增加细胞质静息 $\mathrm{Ca}^{2+}$ 浓度, 使细胞质 处于一种比较容易激发产生钙波的兴奋状态，从而对 全局钙信号的发生产生重要的影响 ${ }^{[37]}$.

\section{2 钙脉冲对钙泡动力学的抑制效应}

进一步, 我们建立了一个随机钻泡模型来讨论静 息钙浓度 $\left[\mathrm{Ca}^{2+}\right]_{\text {Basal }}$ 对钙泡动力学的调控机制. 模型主 要包括一个双钙浓度的决定性方程和一个 $\mathrm{IP}_{3} \mathrm{R}$ 通道打 
开及关闭的随机性过程, 我们讨论了 $\left[\mathrm{Ca}^{2+}\right]_{\text {Basal }}$ 对钙泡 信号的统计学特性的影响 ${ }^{[38]}$. 模型的结果显示, 钻泡 频率和寿命随着 $\left[\mathrm{Ca}^{2+}\right]_{\mathrm{Basal}}$ 的增加而增加.

更重要的是, 我们发现了一个反常的现象: 钙泡的 幅值和打开的最大通道数随着 $\left[\mathrm{Ca}^{2+}\right]_{\text {Basal }}$ 的增加而减小 (图3). 这一反常现象的产生主要由两方面原因造成: 一方面, 由于 $\left[\mathrm{Ca}^{2+}\right]_{\mathrm{Basal}}$ 的增加, 钙泡频率增加, 从而减 少了钙泡的恢复时间; 另一方面，由于钻脉冲信号的频 率增加，钙脉冲的产生会导致通道处于抑制状态，因而 阻止相邻铂泡之间的恢复过程，而随着 $\left[\mathrm{Ca}^{2+}\right]_{\text {Basal }}$ 的增 加，更多的钙脉冲事件的产生导致更多的通道处于抑 制状态，因此只有少数的通道可供打开，导致小幅度的 钙泡信号才能产生. 我们指出, 钻脉冲信号通过对钙泡 信号的抑制调控, 对全局钲波信号产生重要的影响.

\section{4 内质网 IP ${ }_{3}$ R调控的全局钙波动力学}

一个通道集团内的钙泡所释放的 $\mathrm{Ca}^{2+}$ 可能会扩散
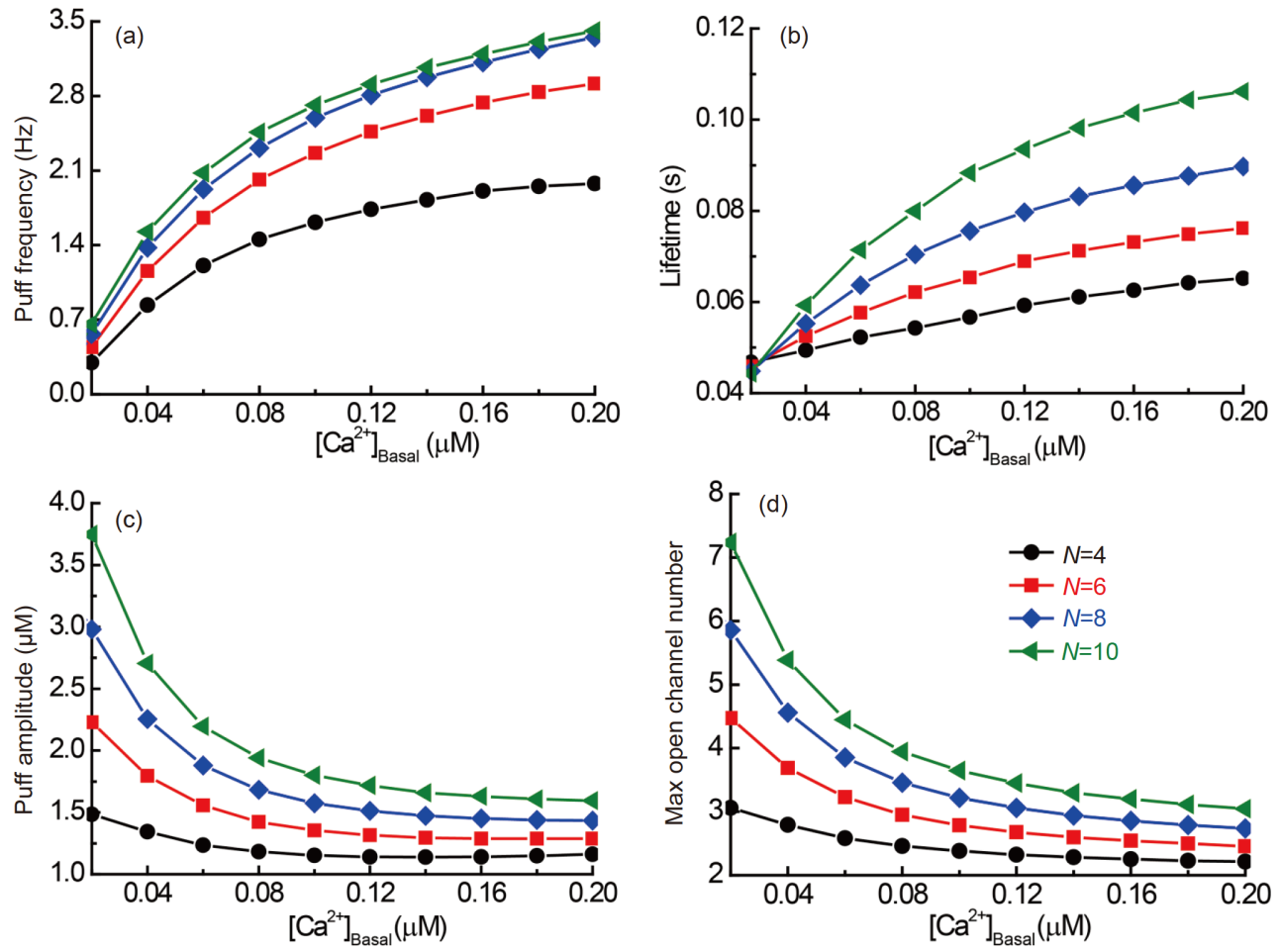

图 3 (网络版彩图)钻泡的频率(a), 寿命(b), 幅值(c) 和打开的最大通道数(d)随 $\left[\mathrm{Ca}^{2+}\right]_{\mathrm{Basal}}$ 的变化情况 ${ }^{[38]}$. 其中, $N=4$ (圆形), 6 (正 方形), 8 (菱形), 10 (三角形)

Figure 3 (Color online) Puff frequency (a), puff lifetime (b), puff amplitude (c), and the maximum open-channel number (d) against $\left[\mathrm{Ca}^{2+}\right]_{\mathrm{Basal}}$ at $N=4$ (circles), 6 (squares), 8 (rhombus), and 10 (triangles) [38]. 
模型研究指出, 局域且短时的钲泡终止动力学, 主 要由高浓度的钙离子结合在钙通道的抑制位点所导 致; 而全局且长时的钙波的终止动力学, 则主要由 $\mathrm{IP}_{3}$ 信号分子在锬通道上的去结合所导致(图4). 我们的研 究揭示了 $\mathrm{IP}_{3}$ 信号分子的一个新作用，展示了细胞如何 充分利用 $I P_{3} R$ 门控通道的复杂特性，得到不同的钻信 号, 从而控制不同的细胞生理功能.

\section{2 钙离子通道集团化分布导致的钲波衰败现象}

集团化钙离子通道, 可以产生多尺度的钻信号, 调 控不同细胞功能. 我们进一步研究如下重要问题: 集团 化分布的离子通道，对全局性钲波是否有特别的调控 作用机制. 我们建立了一个二维钙离子信号扩散反应 模型，其中 $\mathrm{IP}_{3} \mathrm{R}$ 通道在内质网膜上成团簇分布，从而 讨论钙离子信号振荡扩散动力学(图5) ${ }^{[41]}$.

数值模拟表明, 随着钲离子扩散系数增大, 即钻离 子通道耦合增强，集团化分布离子通道导致的钻波出 现一种反常的衰败现象，且扩散波的衰败行为与钻离 子通道动力学是否为确定性或随机性动力学无关. 而 在均匀连续分布的激发扩散系统中，扩散系数的增大， 会导致扩散波越稳定, 不可能出现扩散波的衰败行为.

钙信号控制着多种细胞功能，当细胞内钙离子浓
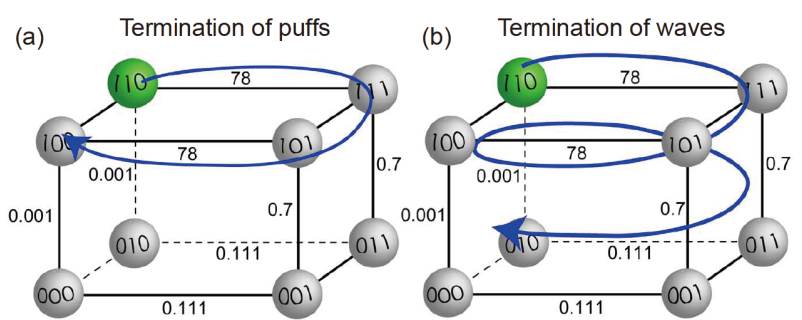

图 4 (网络版彩图)钻通道集团触发钻泡(a)及钻波(b)的终 止动力学行为 ${ }^{[40]}$. (a) 在短暂的钲泡释放期间, 仅在模型的上 平面发生通道的打开/关闭过程; (b) 在钙波过程中, 通道打 开的频率变高, 处于状态111的亚基去结合IP3分子, 并转移 至模型的下平面

Figure 4 (Color online) Illustrate the termination dynamics of subunits during puffs (a) and burst (b) [40]. (a) During short release (puff) only the upper plane of the model is populated by subunits of opening/closing channels; (b) the more frequent the channels open, the subunits in state 111 dissociate IP3 and "cycle" down to the lower plane of the model.

度很高时，会导致细胞的死亡. 所以如何控制钙离子 浓度在适当幅度内，则成为一个重要调控机制。我们 认为，通过集团化通道分布，形成的钲波衰败行为对 钻离子浓度振荡幅度具有重要的限制作用. 我们认为, 扩散波衰败行为，在具有局域化可激发区域的非均匀 扩散系统中, 是一个很普适的行为.

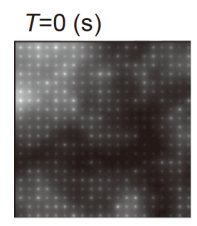

12

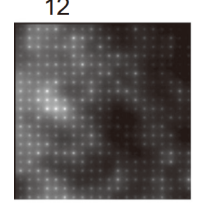

24

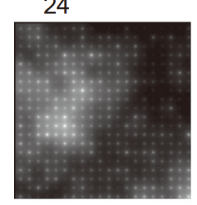

36

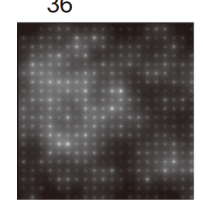

2

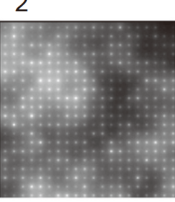

14

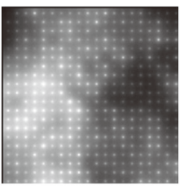

26

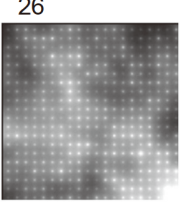

38

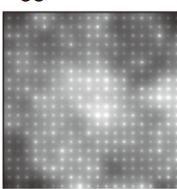

4

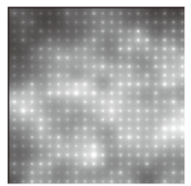

16

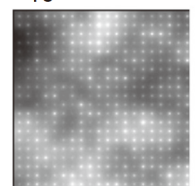

28

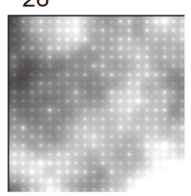

40

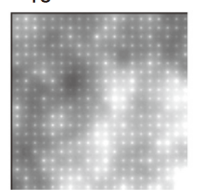

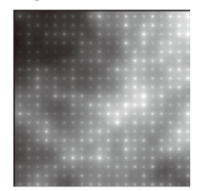

18

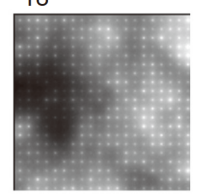

30

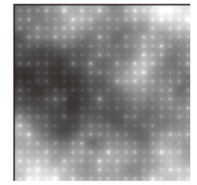

42

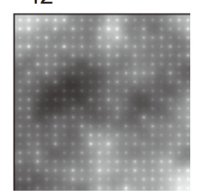

8

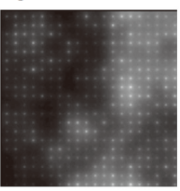

20

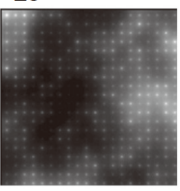

32

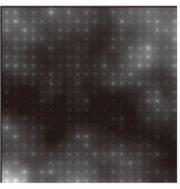

44

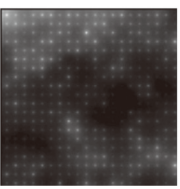

10

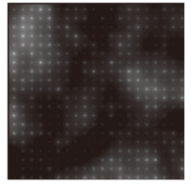

22

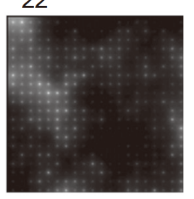

34

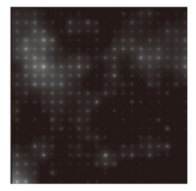

$46(s)$

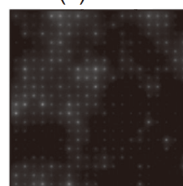

图 5 钙信号扩散波空间随机传播示意图 ${ }^{[4]}$

Figure 5 The snapshots of stochastic spatiotemporal $\mathrm{Ca}^{2+}$ waves propagation [41]. 


\section{5 内质网与线粒体微域钙动力学}

2011年MCU的分子鉴定 ${ }^{[25]}$,使人们对线粒体如何 调控钙信号的研究得到了空前的重视 ${ }^{[26]}$, 我们通过构 建相应的钙信号网络模型，对内质网和线粒体钻信号 间的耦联进行了系统研究, 特别是针对内质网和线粒 体之间的微域尺度, 及其调控作用进行了分析研究.

\section{1 内质网与线粒体间钲微域对钙信号的影响}

内质网与线粒体的空间距离对线粒体摄钙乃至细 胞质钙信号有非常重要的影响. 一方面，由于 $\mathrm{IP}_{3} \mathrm{R}$ 通道 在释放 $\mathrm{Ca}^{2+}$ 时会形成一个随距离迅速衰减的 $\left[\mathrm{Ca}^{2+}\right]$ 梯度, 这两个细胞器之间近距离的接触是钙微域形成的物理 基础. 另一方面, 线粒体是一种机动性很强的细胞器, 细 胞质中 $\left[\mathrm{Ca}^{2+}\right]$ 的提升会使它停止运动 ${ }^{[42,43]}$. 因此, 内质网 与线粒体之间应该存在一个有效的工作距离. 由于实验 手段的限制, 实验上测得的内质网与线粒体之间的距离 范围很大, 小到不足 $10 \mathrm{~nm}$, 大到超过 $200 \mathrm{~nm}^{[44,45]}$.

为阐明内质网与线粒体之间的有效工作距离, 我 们构建了内质网与线粒体利用钲微域耦合的钙信号动 力学模型(图6) ${ }^{[46]}$. 该研究通过对 $\mathrm{Ca}^{2+}$ 信号振荡动力学 的讨论, 预测了内质网与线粒体之间的距离约为 10-65 nm. 这一距离与最新实验测量数据 10-80 nm高 度吻合 ${ }^{[47]}$. 因此，该钲微域模型为涉及内质网与线粒 体距离方面的研究提供了理论基础.

\section{2 钙连蛋白对内质网钙洜的促进作用}

钙连蛋白(Calnexin)是一种位于内质网膜上的蛋白 质, 除了参与蛋白折叠这一主要功能外, 它还可与内质网

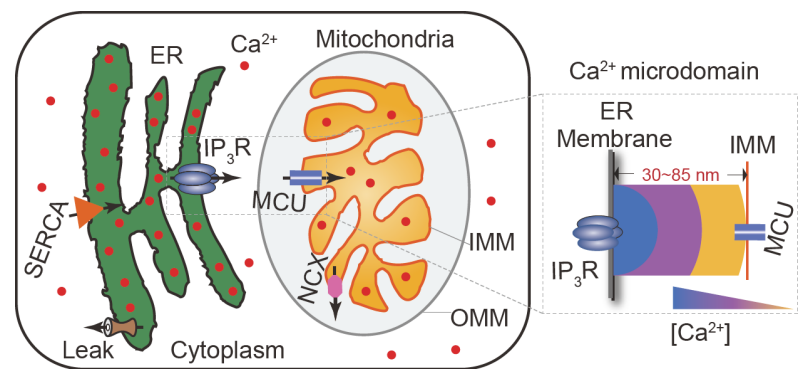

图 6 (网络版彩图)内质网与线粒体利用钲微域相互耦合的 钲信号模型示意图 ${ }^{[46]}$

Figure 6 (Color online) The schematic diagram of the $\mathrm{Ca}^{2+}$ dynamic model that coupling the crosstalk between endoplasime reticulum and mitochondria by microdomain [46].
上的 $\mathrm{Ca}^{2+}$ 原SERCA相互作用, 从而调节钙振荡. 早期的研 究发现钻连蛋白对钲振荡有抑制作用 ${ }^{[48]}$, 最新研究指出 钙连蛋白对SERCA有促进作用. 为此, 我们理论结合实 验, 利用钙微域模型证实, 钙连蛋白可通过促进SERCA 的活性从而减少细胞质中的 $\mathrm{Ca}^{2+}$ 浓度, 导致内质网与线 粒体之间距离增加. 在此基础上，我们进一步阐明了钙 连蛋白影响线粒体产生能量的分子机制 ${ }^{[49]}$.

\section{6 病理情况下钙信号和细胞信号网络动 力学}

钙信号和蛋白信号网络参与各种细胞功能调控, 而非正常的钲离子浓度和异常信号网络动力学则与各 种疾病密切相关. 阿尔茨海默症(Alzheimer's Disease, $\mathrm{AD})$ 是一种常见的神经元遭受缓慢破坏并引起严重认 知障碍的慢性疾病 ${ }^{[50]}$, 它的发生与 $I P_{3} \mathrm{R}$ 调控的钻信号 也有着密切的关联, 由激动剂 $I \mathrm{I}_{3}$ 介导的 $\mathrm{Ca}^{2+}$ 信号的增 强已经作为一种特征用于诊断家族性阿尔茨海默症. 我们进一步利用模型, 讨论了病理情况下钙信号和细 胞信号网络动力学行为.

\section{1 阿尔茨海默症中 $I P_{3} R$ 通道动力学}

我们首次从理论模型上研究了细胞早老素突变如 何导致通道动力学的变化, 从而致使阿尔茨海默症患 者细胞中钙信号传导失调的动力学机制. 基于生物膜 片钳实验结果, 通过对 $I P_{3} \mathrm{R}$ 通道的开关动力学进行建 模, 我们对比讨论了正常细胞和家族性阿尔茨海默症 (FAD) 染病细胞中的 $I P_{3} \mathrm{R}$ 通道的打开概率、平均打开 时间和平均关闭时间 ${ }^{[51]}$.

我们发现，FAD染病细胞的离子通道打开概率明 显增大的主要原因是变异的 $\mathrm{M} 146 \mathrm{~L}$ 提高了 $\mathrm{IP}_{3} \mathrm{R}$ 对激动 剂 $I P_{3}$ 和激活钙的亲和力. 该建模研究为探索早老素突 变导致家族性阿尔茨海默症的机制提供了一个理论 依据。

\section{2 高浓度钲信号可导致阿尔茨海默症}

基于钲微域模型中的理论结果, 结合大量实验事实, 我们提出了“内质网与线粒体之间距离的减少通过增强 的钙信号引发阿尔茨海默症”的信号网络模型假说 ${ }^{[52]}$.

该假说认为, 细胞各种各样的老化病因会使得内 质网与线粒体之间的距离减小, 从而导致细胞质与线 
粒体中的钙离子同时过载. 一方面, 细胞质钙离子过载 可以(1) 过分激活依赖钙离子的酶，这些酶通过调节 $\mathrm{Bcl}-2$ 蛋白质家族的活性, 导致线粒体外膜通透成孔, 细 胞色素c通过此孔释放出来，激活可破坏体内多种蛋白 质的Caspase-3蛋白; (2) 通过激活Caspase-12间接激活 Caspase-3; (3) 促进 $\beta$-淀粉样蛋白的生成和聚合. 这三个 途径都会引发神经细胞调亡. 另一方面, 线粒体钻离子 过载会增加活性氧的生成, 诱导线粒体通透性转换孔开 放，促使神经元调亡和坏死同时发生. 负责记忆和认知 功能的神经元若大量死亡(包括调亡和坏死), 就会引发 阿尔茨海默症. 因此, 我们的假说包含了目前流行的4种 阿尔茨海默症发病机制学说中提到的主要元素, 为更全 面地了解该病的病理学提供了新的视角.

\section{3 细胞凋亡信号网络揭示同义突变及癌症发生 的关联性}

长期以来, 由于同义突变不影响所编码的蛋白质 产物, 一直不被人们所关注 ${ }^{[33,54]}$. 然而, 近些年的一些 研究表明, 同义突变可能通过诸多生理过程对细胞的
正常功能产生影响, 引起疾病的发生. 我们基于 $\mathrm{TNF}-\alpha$ 诱导的细胞存活及调亡信号网络模型 ${ }^{[55]}$, 指出介导细 胞存活和调亡的TNF- $\alpha$ 关键剂量，而基因突变会通过 影响信号网络中蛋白反应过程, 改变信号网络的TNF$\alpha$ 关键剂量, 从而影响细胞的生死.

我们进一步建立了 TNF- $\alpha$ 关键剂量参数的敏感性 与基因突变的关联性. 基于该关联性, 我们分别研究了 9种不同癌症中, 基因的无义突变、错义突变以及同义 突变与对应模型参数敏感性的关联性(图7). 结果表明, 通过引入同义突变，9种癌症中的6种对应基因的突变 数据与模型的参数敏感性具有很好的相关性, 另外 3 种呈现出类似的相关性. 上述结果说明，除了错义和 无义两种类型的突变，同义突变对于癌症发生的作用 不能被忽视.

\section{7 钲信号和细胞死亡信号网络动力学}

钙信号及信号网络的异常, 不仅能引起疾病的发 生，在细胞死亡方面同样扮演着重要的角色. 针对钲
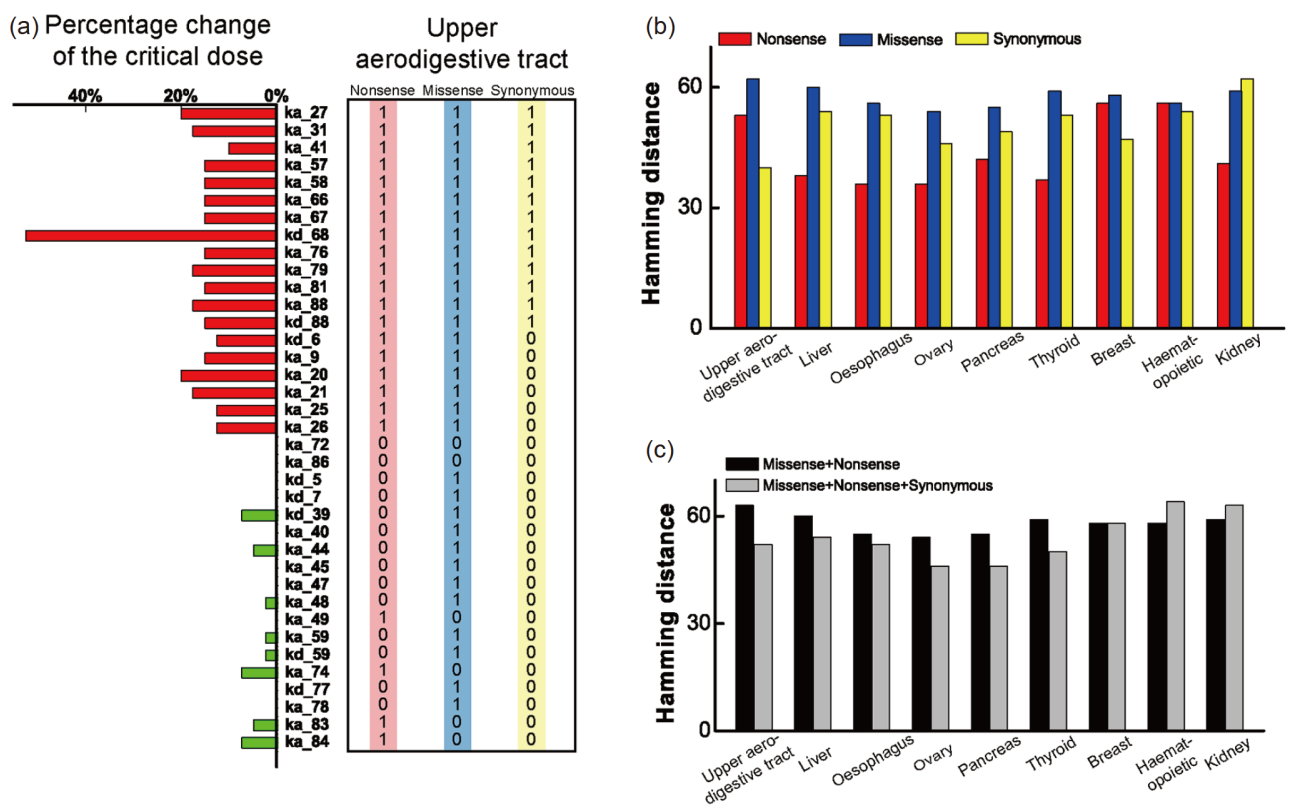

图 7 (网络版彩图)调亡信号网络参数敏感性与不同癌症的关联性分析 ${ }^{[55]}$. (a) 参数敏感性与上消化道癌基因突变数据的比 较; (b) 参数敏感性与9种不同癌症基因突变数据的汉明距离; (c) 参数敏感性与分别考虑两种和三种类型基因突变的癌症突 变数据的汉明距离

Figure 7 (Color online) Analysis of correlation between apoptosis network parameter sensitivity and the corresponding cancer-related mutations [55]. (a) Comparison between parameter sensitivity and the corresponding genes mutations of upper aerodigestive tract cancer; (b) calculated hamming distances between parameter sensitivity and the genes spectra for genes mutations of 9 cancers; (c) calculated hamming distances between parameter sensitivity and the genes mutations with combined action of the two and three mutations, respectively. 
信号和细胞信号网络动力学调控细胞死亡发生这一重 要生物学问题, 我们也进行了系统的动力学建模研究.

\subsection{Bcl-2蛋白抑制钙信号的动力学分析}

Bcl-2蛋白是参与细胞调亡的重要蛋白, 可直接或 间接调控 $I P_{3} \mathrm{R}$ 钙信号通道的活性，影响钙信号的频率 和振幅, 从而决定细胞的命运 ${ }^{[6,57]}$. 我们构建了 Bcl-2 蛋白调控 $\mathrm{Ca}^{2+}$ 的信号通路的网络模型 ${ }^{[58]}$, 重现了相关 实验结果, 并从分岔参数的角度对模型进行了系统的 分析，证明了 Bcl-2蛋白对钙信号的抑制作用，Bcl-2对 刺激强度能产生 $\mathrm{Ca}^{2+}$ 振荡的区域有重要影响, 且PP1可 以有效地抑制钙信号，而PKA对钙信号的促进作用有 一定的局限性. 该结果不仅对相关生物学实验有一定 的指导作用，而且为治疗因钙信号失调而导致的疾病 提供思路.

\section{2 细胞凋亡体自组装最佳途径讨论}

细胞调亡的内途径主要依赖于调亡体Apoptosome的形成 ${ }^{[59]}$. 当细胞受到调亡刺激时, 位于线粒体 膜间隙的细胞色素c (Cyt c) 释放到细胞质中，与单体 形式的调亡蛋白酶活化因子1 (Apaf-1)结合, 引起 Apaf-1构象改变，随后7个Apaf-1·Cyt c复合物(AC)组 装成“车轮状”的凋亡体. 调亡体形成后引发Caspase级 联反应, 最终导致细胞调亡.

为了阐明 7 个 $\mathrm{AC}$ 复合物如何自组装成凋亡体这一
重要问题, 我们构建了一个调亡体自组装反应模型 ${ }^{[60]}$, 指出从一个 $\mathrm{AC}$ 单体组装成七聚体的调亡体有 11 条基 本路径, 并列举了由它们任意组合而成的2047种组装 途径(图8). 通过数学分析和数值模拟, 我们发现了两 条最优基本路径，并结合实验观测结果，综合考虑转 化效率和速度两项指标，找到52种最优组装途径. 该 研究不仅首次从理论方面对调亡体的组装过程进行了 全面分析，而且可为生物体内存在众多的多聚体的组 装过程提供研究思路.

\subsection{TLR4复合物应激LPS刺激的自组装策略}

脂多糖(LPS)是细菌细胞膜外膜的主要成分, 通过 细胞受体TLR4的识别, 进而激活细胞内的免疫、死亡 等关键信号通路 ${ }^{[61]}$. TLR4的复合物主要包含TLR4, TIRAP，MyD88，IRAK1/4和TRAF6等蛋白. 针对目前 对TLR4复合物组装的动力学过程尚不清楚, 我们基于 最新的质谱数据, 构建了描述TLR4复合物组装过程的 数学模型 ${ }^{[62]}$, 对TLR4复合物自组装动力学进行研究.

通过模拟分析，我们发现在LPS刺激下，存在不依 赖于TIRAP的MyD88复合物的自组装，且该复合物不 包括TRAF6 (图9)。进一步模拟发现，TIRAP依赖和 TIRAP不依赖的两种复合物的组装行为都依赖于LPS 的刺激强度. 为了更有效的信号转导，低刺激下 MyD88会形成更长的链状结构. 这种链状结构同时也 受复合物中相关蛋白的浓度调控. 该研究加深了我们

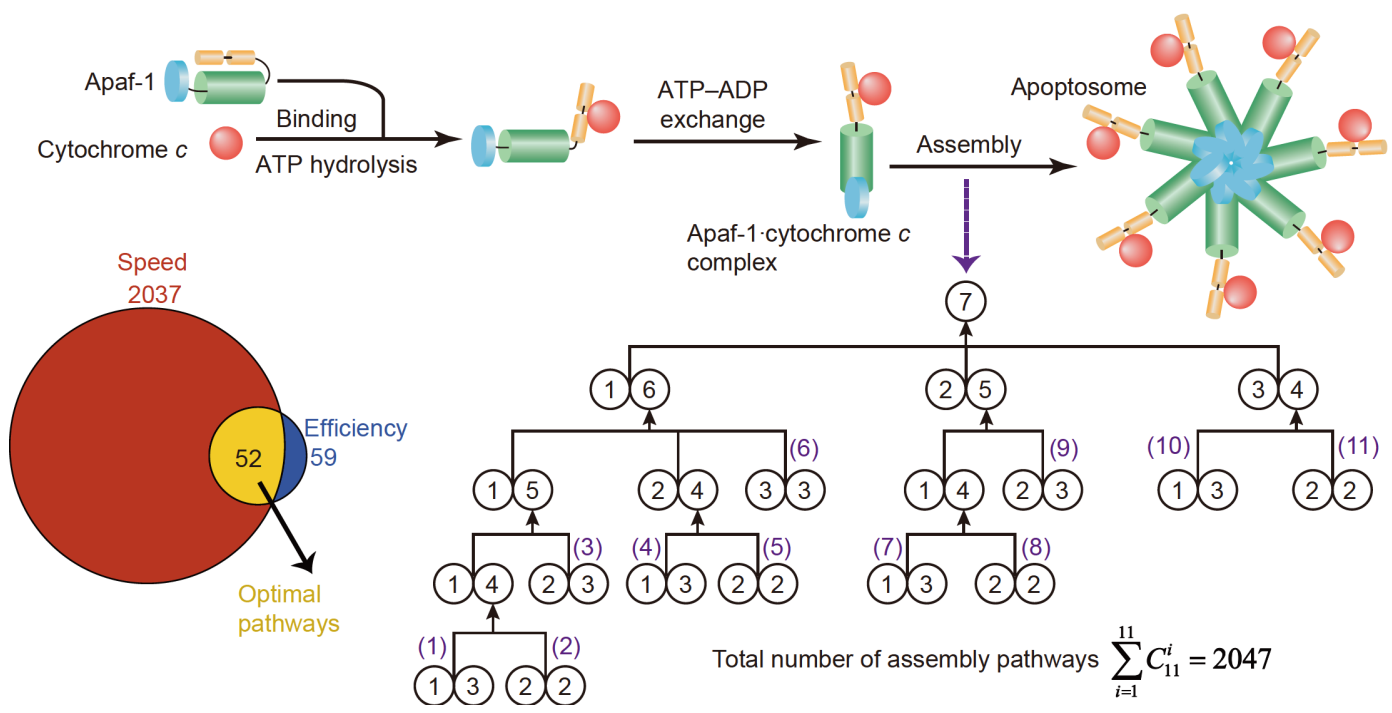

图 8 (网络版彩图)调亡复合体形成的过程及11条基本路径 ${ }^{[60]}$

Figure 8 (Color online) Mechanism of apoptosome formation and all 11 elementary routes for apoptosome assembly [60]. 


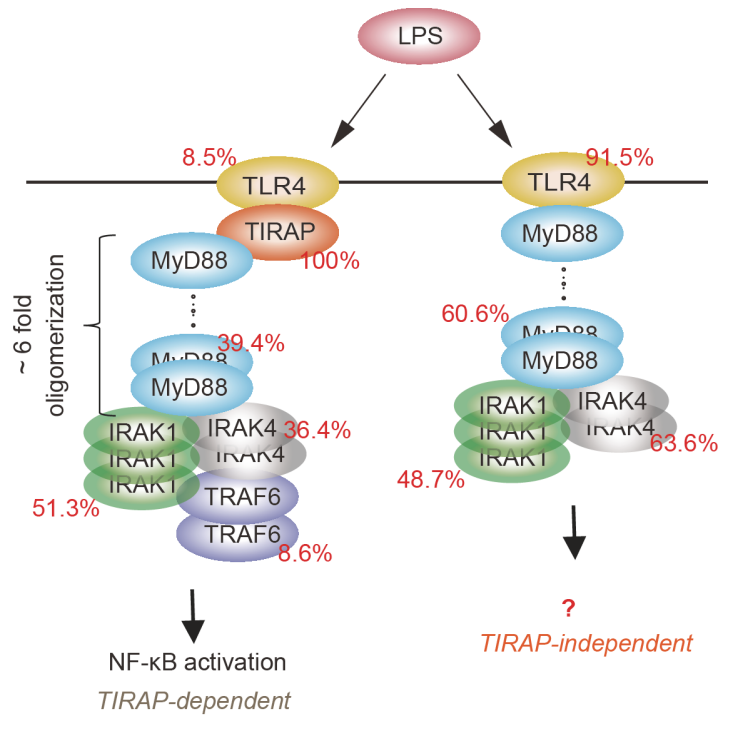

图 9 (网络版彩图) LPS介导的TLR4复合物的主要成分及 分布 ${ }^{[62]}$

Figure 9 (Color online) Schematic diagram of the components distribution in LPS-mediated TLR4 complexes [62].
对TLR4复合物自组装过程的理解, 并预测了新的复合 物存在，为细胞死亡等不同响应策略提供了进一步的 理论解释.

\section{4 细胞钙信号调控细胞凋亡的动力学机制}

尽管早在20年前, 研究者们已发现细胞质中钻浓 度的异常升高会导致细胞调亡，但由于实验上很难同 时监测快速变化的钙信号和缓慢变化的调亡蛋白，导 致有两个基本问题悬而未决：一是振荡的钻信号如何 转化为类似全或无的细胞命运响应，二是钙信号到底 是通过调幅还是调频的方式调控细胞调亡.

为此我们构建了细胞质钲信号调控细胞调亡信号 网络模型(图 10) ${ }^{[63]}$, 该模型包含 4 个模块：钙振荡模 块、钻调蛋白模块、Bcl-2蛋白家族控制线粒体外膜 通透(MOMP)模块、Caspase级联反应模块. 模拟结果 显示振荡的钙信号虽然能传递到钙调蛋白模块，但在 经过Bcl-2蛋白家族控制MOMP模块时逐渐转化为渐

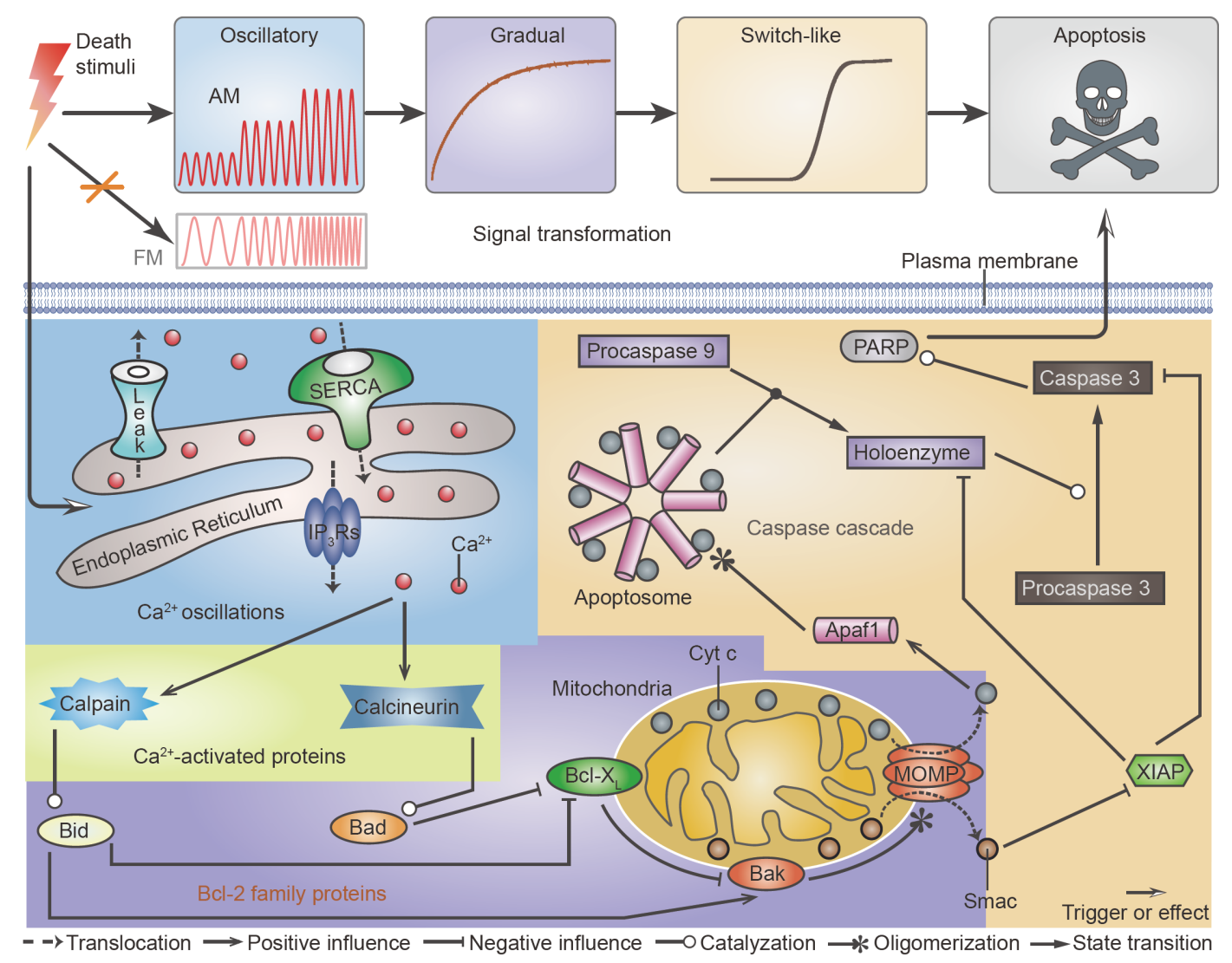

图 10 (网络版彩图)细胞质钙信号调控细胞调亡的信号网络 ${ }^{[63]}$

Figure 10 (Color online) The role of cytoplasmic $\mathrm{Ca}^{2+}$ in regulating apoptosis signaling network [63]. 
变信号, 最终到达Caspase级联反应模块时, 转变为类 似全或无的阈值跳变信号. 在信号传递过程中, 钙信号 的振幅属性可以很好地传递到下游，但其频率属性在 中途被屏蔽，导致细胞调亡对钙信号的振幅变化很敏 感,但对其频率变化不敏感. 因此, 该研究表明钻信号 是以调幅的方式调控细胞调亡.

\section{5 细胞凋亡和细胞坏死间的抉择动力学机制}

细胞凋亡和细胞坏死是细胞最主要的两种死亡方 式 ${ }^{[64]}$ ，当前对这两种死亡方式之间通路串扰及抉择机 制的定量理解上仍存在很大挑战. 我们研究表明蛋白 信号RIP1，pro-caspase-8和RIP3的定量相互作用在该 通路串扰中起关键作用 ${ }^{[65]}$. 结合质谱技术SWATH-MS 测量结果的精准建模和L929纤维肉瘤细胞的实验验 证，我们发现当RIP1在细胞中的表达水平低于每细胞 约1000个分子 $(\mathrm{mpc})$ 时，该细胞仅发生TRADD依赖性 细胞凋亡. 当RIP1水平高于约 $1000 \mathrm{mpc}$ 时, pro-caspase-8和RIP3分别被募集到坏死小体，对RIP1表达量 具有线性和非线性依赖关系的动力学响应, 这很好地 解释了细胞凋亡和坏死过程的同时发生, 以及悖论性 的传统认知, 细胞坏死需要RIP1, 但它的增加下调了 坏死过程. 较高的RIP1表达(>46000 mpc)抑制调亡, 仅 导致细胞坏死. 所以, RIP1表达水平双相调控细胞死 亡和细胞坏死. 本研究定量阐述了坏死小体中组分相 互作用的程度和动力学模式对于细胞命运的抉择至关 重要, 为后续精准定量研究生命系统的动力学行为及 调控机制提供了方向.

\section{8 总结与展望}

$\mathrm{Ca}^{2+}$ 是细胞内广泛存在的一种重要的第二信使,
参与并控制着几乎所有的生命活动过程. 大部分真核 细胞中细胞质 $\mathrm{Ca}^{2+}$ 浓度复杂的时空动力学都依赖于内 质网和线粒体这两种主要的细胞器. 由于钙离子信号 是生物系统中不可或缺的重要信号, 几乎参与了所有 的生命过程. 因此, 现在对于钙信号的理论研究也进 入了更深层次. 近年来, 我们主要聚焦于内质网随机 钙信号动力学以及内质网与线粒体相互耦合的钙信号 动力学, 并阐明了它们在生理功能或生命现象中的作 用. 我们着重研究了 $\mathrm{Ca}^{2+}$ 在细胞调亡中的作用, 并以此 为出发点研究了一些与细胞命运抉择相关的信号调控 网络, 此外还探究了它们与癌症和阿尔兹海默症的 关系.

关于细胞死亡的分子机制是近些年以及未来一段 时期的研究焦点, 各种细胞死亡方式, 如调亡、自噬、 焦亡、坏死等的信号网络及其相互关系的阐明，均依 赖于动力学机制方面的研究. 除了 $\mathrm{Ca}^{2+}$ 之外, 还有一些 其他物质, 如Bcl-2等蛋白家族 ${ }^{[66]}$, 在这些信号网络中 发挥关键作用, 关于它们的研究必将推动细胞死亡领 域的发展.

人们通过对细胞信号网络进行生物物理建模，开 展定量计算和动力学分析研究, 发展了一系列理论和 方法. 而如何综合运用非平衡统计物理的理论、非线 性动力学手段, 包括用能量景观、能量耗散理论和信

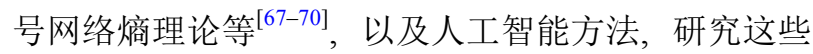
细胞信号网络动力学和调控机制, 以及相关物理特性, 则是当前信号网络动力学理论建模的重要挑战. 我们 相信，通过这些理论研究，将进一步揭示细胞信号网 络的非线性动力学及其非平衡统计物理特性, 为细胞 信号网络生物功能的理解及其调控机制提供坚实的理 论基础, 为推动人类健康的策略与生命科学的发展提 供交叉学科学术视野.

\section{参考文献}

1 Berridge M J, Bootman M D, Roderick H L. Calcium signalling: Dynamics, homeostasis and remodelling. Nat Rev Mol Cell Biol, 2003, 4: 517529

2 Berridge M J, Bootman M D, Lipp P. Calcium-A life and death signal. Nature, 1998, 395: 645-648

3 Orrenius S, Zhivotovsky B, Nicotera P. Regulation of cell death: The calcium-apoptosis link. Nat Rev Mol Cell Biol, 2003, 4: 552-565

4 Rizzuto R, De Stefani D, Raffaello A, et al. Mitochondria as sensors and regulators of calcium signalling. Nat Rev Mol Cell Biol, 2012, 13: 566578

5 Cheng H, Lederer W J, Cannell M B. Calcium sparks: Elementary events underlying excitation-contraction coupling in heart muscle. Science, 1993, 262: 740-744 
6 Gilkey J C, Jaffe L F, Ridgway E B, et al. A free calcium wave traverses the activating egg of the medaka, Oryzias latipes. J Cell Biol, 1978, 76: $448-466$

7 Dupont G, Houart G, Goldbeter A. From simple to complex $\mathrm{Ca}^{2+}$ oscillations: Regulatory mechanisms and theoretical models. In: Understanding Calcium Dynamics. Berlin: Springer, 2003. 401-405

8 Lechleiter J, Girard S, Peralta E, et al. Spiral calcium wave propagation and annihilation in Xenopus laevis oocytes. Science, 1991, 252: 123-126

9 Lechleiter J D, John L M, Camacho P. $\mathrm{Ca}^{2+}$ wave dispersion and spiral wave entrainment in Xenopus laevis oocytes overexpressing $\mathrm{Ca}^{2+}$ ATPases. Biophys Chem, 1998, 72: 123-129

10 Nuccitelli R, Yim D L, Smart T. The sperm-induced $\mathrm{Ca}^{2+}$ wave following fertilization of the xenopus egg requires the production of Ins $(1,4,5) \mathrm{P}_{3}$. Dev Biol, 1993, 158: 200-212

11 Wagner J, Li Y X, Pearson J, et al. Simulation of the fertilization $\mathrm{Ca}^{2+}$ wave in Xenopus laevis eggs. Biophys J, 1998, 75: 2088-2097

12 Verkhratsky A, Toescu E C. Endoplasmic reticulum $\mathrm{Ca}^{2+}$ homeostasis and neuronal death. J Cell Mol Med, 2003, 7: 351-361

13 Taylor C W, Tovey S C. IP3 receptors: Toward understanding their activation. Cold Spring Harbor Perspect Biol, 2010, 2: a004010

14 Yao Y, Choi J, Parker I. Quantal puffs of intracellular $\mathrm{Ca}^{2+}$ evoked by inositol trisphosphate in Xenopus oocytes. J Physiol, 1995, 482: 533-553

15 Sun X P, Callamaras N, Marchant J S, et al. A continuum of Ins $\mathrm{P}_{3}$-mediated elementary $\mathrm{Ca}^{2+}$ signalling events in Xenopus oocytes. J Physiol, 1998, 509: 67-80

16 Rose H J, Dargan S, Shuai J, et al. 'Trigger' events precede calcium puffs in Xenopus oocytes. Biophys J, 2006, 91: 4024-4032

17 Shuai J, Rose H J, Parker I. The number and spatial distribution of $\mathrm{IP}_{3}$ receptors underlying calcium puffs in Xenopus oocytes. Biophys J, 2006, 91: 4033-4044

18 Berridge M J. Elementary and global aspects of calcium signalling. J Physiol, 1997, 499: 291-306

19 Rizzuto R, Brini M, Murgia M, et al. Microdomains with high $\mathrm{Ca}^{2+}$ close to $\mathrm{IP}_{3}$-sensitive channels that are sensed by neighboring mitochondria. Science, 1993, 262: 744-747

20 Rizzuto R, Pinton P, Carrington W, et al. Close contacts with the endoplasmic reticulum as determinants of mitochondrial Ca ${ }^{2+}$ responses. Science, 1998, 280: 1763-1766

21 Dai J, Kuo K H, Leo J M, et al. Rearrangement of the close contact between the mitochondria and the sarcoplasmic reticulum in airway smooth muscle. Cell Calcium, 2005, 37: 333-340

22 Csordas G, Renken C, Varnai P, et al. Structural and functional features and significance of the physical linkage between ER and mitochondria. J Cell Biol, 2006, 174: 915-921

23 Csordás G, Várnai P, Golenár T, et al. Imaging interorganelle contacts and local calcium dynamics at the ER-mitochondrial interface. Mol Cell, 2010, 39: 121-132

24 Giacomello M, Drago I, Bortolozzi M, et al. $\mathrm{Ca}^{2+}$ hot spots on the mitochondrial surface are generated by $\mathrm{Ca}^{2+}$ mobilization from stores, but not by activation of store-operated $\mathrm{Ca}^{2+}$ channels. Mol Cell, 2010, 38: 280-290

25 De Stefani D, Raffaello A, Teardo E, et al. A forty-kilodalton protein of the inner membrane is the mitochondrial calcium uniporter. Nature, 2011, 476: $336-340$

26 Zhuo W, Zhou H, Guo R, et al. Structure of intact human MCU supercomplex with the auxiliary MICU subunits. Protein Cell, 2020

27 Vermassen E, Van Acker K, Annaert W G, et al. Microtubule-dependent redistribution of the type-1 inositol 1,4,5-trisphosphate receptor in A7r5 smooth muscle cells. J Cell Sci, 2003, 116: 1269-1277 Wilson B S, Pfeiffer J R, Smith A J, et al. Calcium-dependent clustering of inositol 1,4,5-trisphosphate receptors. MBoC, 1998, 9: 1465-1478 Chalmers M, Schell M J, Thorn P. Agonist-evoked inositol trisphosphate receptor $\left(\mathrm{IP}_{3} \mathrm{R}\right)$ clustering is not dependent on changes in the structure of the endoplasmic reticulum. Biochem J, 2006, 394: 57-66

30 Boulware $\mathrm{M} \mathrm{J}$, Marchant $\mathrm{J} \mathrm{S}$. $\mathrm{IP}_{3}$ receptor activity is differentially regulated in endoplasmic reticulum subdomains during oocyte maturation. Curr Biol, 2005, 15: 765-770

31 Wei F, Yang D, Straube R, et al. Brownian diffusion of ion channels in different membrane patch geometries. Phys Rev E, 2011, 83: 021919 Amicarelli F, Colafarina S, Cesare P, et al. Morphofunctional mitochondrial response to methylglyoxal toxicity in Bufo bufo embryos. Int J Biochem Cell Biol, 2001, 33: 1129-1139

33 Rüdiger S, Shuai J W, Huisinga W, et al. Hybrid stochastic and deterministic simulations of calcium blips. Biophys J, 2007, 93: 1847-1857

34 Rüdiger S, Nagaiah C, Warnecke G, et al. Calcium domains around single and clustered $\mathrm{IP}_{3}$ receptors and their modulation by buffers. Biophys $\mathrm{J}$, 
2010, 99: 3-12 Sci USA, 2009, 106: 6404-6409

40

4 012406

42 Yi M, Weaver D, Hajnoczky G. Control of mitochondrial motility and distribution by the calcium signal. J Cell Biol, 2004, 167: 661-672

43 Wang X, Schwarz T L. The mechanism of $\mathrm{Ca}^{2+}$-dependent regulation of kinesin-mediated mitochondrial motility. Cell, 2009, 136: 163-174

44 de Brito O M, Scorrano L. An intimate liaison: Spatial organization of the endoplasmic reticulum-mitochondria relationship. EMBO J, 2010, 29: $2715-2723$

45 Marchi S, Patergnani S, Pinton P. The endoplasmic reticulum-mitochondria connection: One touch, multiple functions. Biochim Biophys Acta, 2013, 1837: 461-469

46 Qi H, Li L, Shuai J. Optimal microdomain crosstalk between endoplasmic reticulum and mitochondria for $\mathrm{Ca}^{2+}$ oscillations. Sci Rep, 2015, 5: 7984

47 Giacomello M, Pellegrini L. The coming of age of the mitochondria-ER contact: A matter of thickness. Cell Death Differ, 2016, 23: 1417-1427

Roderick H L, Lechleiter J D, Camacho P. Cytosolic phosphorylation of calnexin controls intracellular $\mathrm{Ca}^{2+}$ oscillations via an interaction with SERCA2b. J Cell Biol, 2000, 149: 1235-1248

49 Gutiérrez T, Qi H, Yap M C, et al. The ER chaperone calnexin controls mitochondrial positioning and respiration. Sci Signal, 2020, 13: eaax6660

50 Hutton M, Hardy J. The presenilins and Alzheimer's disease. Human Mol Genets, 1997, 6: 1639-1646

51 Wei F, Li X, Cai M, et al. Regulation of 1,4,5-triphosphate receptor channel gating dynamics by mutant presenilin in Alzheimer's disease cells. Front Phys, 2017, 12: 128702

52 Qi H, Shuai J. Alzheimer's disease via enhanced calcium signaling caused by the decrease of endoplasmic reticulum-mitochondrial distance. Med Hypotheses, 2016, 89: 28-31

53 Chamary J V, Parmley J L, Hurst L D. Hearing silence: Non-neutral evolution at synonymous sites in mammals. Nat Rev Genet, 2006, 7: 98-108

54 Plotkin J B, Kudla G. Synonymous but not the same: The causes and consequences of codon bias. Nat Rev Genet, 2011, 12: 32-42

55 Li X, Chen Y, Qi H, et al. Synonymous mutations in oncogenesis and apoptosis versus survival unveiled by network modeling. Oncotarget, 2016, 7: 34599-34616

56 Rong Y P, Bultynck G, Aromolaran A S, et al. The BH4 domain of Bcl-2 inhibits ER calcium release and apoptosis by binding the regulatory and coupling domain of the $\mathrm{IP}_{3}$ receptor. Proc Natl Acad Sci USA, 2009, 106: 14397-14402

57 Chang M J, Zhong F, Lavik A R, et al. Feedback regulation mediated by Bcl-2 and DARPP-32 regulates inositol 1,4,5-trisphosphate receptor phosphorylation and promotes cell survival. Proc Natl Acad Sci USA, 2014, 111: 1186-1191

58 Niu S, Shuai J W, Qi H. Modeling of Bcl-2 protein suppressed calcium signaling and its global dynamics analysis (in Chinese). Acta Phys Sin, 2017, 66: 238701 [牛帅, 帅建伟, 祁宏. Bcl-2蛋白抑制钲信号的建模与全局动力学分析. 物理学报, 2017, 66: 238701]

59 Zhou M, Li Y, Hu Q, et al. Atomic structure of the apoptosome: Mechanism of cytochrome $c$ - and dATP-mediated activation of Apaf-1. Genes Dev, 2015, 29: 2349-2361

60 Qi H, Jiang Y, Yin Z, et al. Optimal pathways for the assembly of the Apaf-1·cytochrome $c$ complex into apoptosome. Phys Chem Chem Phys, 2018, 20: 1964-1973

61 Beutler B, Rietschel E T. Innate immune sensing and its roots: The story of endotoxin. Nat Rev Immunol, 2003, 3: 169-176

62 Li X, Zhong C Q, Yin Z, et al. Data-driven modeling identifies TIRAP-independent MyD88 activation complex and myddosome assembly strategy in LPS/TLR4 signaling. Int J Mol Sci, 2020, 21: 3061 
63 101671

64 Mol Cell Biol, 2014, 15: 135-147

Li X, Zhong C Q, Wu R, et al. RIP1-dependent linear and nonlinear recruitments of caspase-8 and RIP3 respectively to necrosome specify distinct cell death outcomes. Protein Cell, 2021, doi: 10.1007/s13238-020-00810-X Cell Biol, 2019, 41: 1127-1134 [许国平, 杨鹏, 祁宏. Bcl-2蛋白家族调节调亡和自潄信号通路的研究进展. 中国细胞生物学学报, 2019, 41: 1127-1134]

67 Wang J, Li C, Wang E. Potential and flux landscapes quantify the stability and robustness of budding yeast cell cycle network. Proc Natl Acad Sci USA, 2010, 107: 8195-8200

Qian H, Bishop L M. The chemical master equation approach to nonequilibrium steady-state of open biochemical systems: Linear singlemolecule enzyme kinetics and nonlinear biochemical reaction networks. Int J Mol Sci, 2010, 11: 3472-3500

69

Zhang D, Cao Y, Ouyang Q, et al. The energy cost and optimal design for synchronization of coupled molecular oscillators. Nat Phys, 2020, 16: 95-100, arXiv: 2001.10479

\title{
Dynamics of calcium signals and the intracellular regulatory signaling network
}

\author{
LI Xiang ${ }^{1}$, QI Hong ${ }^{2}$, HUANG YanDong ${ }^{3} \&$ SHUAI JianWei ${ }^{1 *}$ \\ ${ }^{1}$ Fujian Provincial Key Lab for Soft Functional Materials Research, Research Institute for Biomimetics and Soft Matter, \\ Department of Physics, College of Physical Science and Technology, Xiamen University, Xiamen 361005, China; \\ ${ }_{2}^{2}$ Complex Systems Research Center, Shanxi University, Taiyuan 030006, China; \\ ${ }^{3}$ College of Computer Engineering, Jimei University, Xiamen 361021, China
}

The calcium ion $\left(\mathrm{Ca}^{2+}\right)$ acts as a ubiquitous cellular messenger, regulating a wide variety of cellular processes. The intracellular signaling network precisely controls the normal and pathophysiological functions of cells to conduct various physiological activities. This article reviews our research progress on the dynamics of intracellular $\mathrm{Ca}^{2+}$ signaling and cellular signaling network models in recent years, including the release of local $\mathrm{Ca}^{2+}$ signals from clustered $\mathrm{Ca}^{2+}$ channels, global $\mathrm{Ca}^{2+}$ waves in cells, $\mathrm{Ca}^{2+}$ regulation with microdomains between the endoplasmic reticulum and mitochondria, dynamics of cell apoptosis regulated by $\mathrm{Ca}^{2+}$ signaling, and dynamics of cellular signaling regulation networks. These simulation studies provide directions and ideas for future research on how $\mathrm{Ca}^{2+}$ signals and cellular signaling networks regulate the complicated life functions in cells.

\section{$\mathrm{Ca}^{2+}$ signaling, endoplasmic reticulum, $\mathrm{IP}_{3} \mathrm{R}$ channel, cell death, signaling network dynamics}

PACS: $87.16 . \mathrm{Vy}, 87.50 . \mathrm{cf}, 87.17 . \mathrm{Aa}$

doi: $10.1360 /$ SSPMA-2020-0442 